Research Article

\title{
Human-Machine Cooperation and Path Planning for Complex Road Conditions
}

\author{
Guanghong Zhou (iD) \\ Department of Control Technology, Wuxi Institute of Technology, Wuxi, Jiangsu 214121, China \\ Correspondence should be addressed to Guanghong Zhou; zhough@wxit.edu.cn
}

Received 19 May 2021; Revised 17 June 2021; Accepted 28 June 2021; Published 6 July 2021

Academic Editor: Chenxi Huang

Copyright (C) 2021 Guanghong Zhou. This is an open access article distributed under the Creative Commons Attribution License, which permits unrestricted use, distribution, and reproduction in any medium, provided the original work is properly cited.

\begin{abstract}
With the rapid development of the information age, the development of industrial robots is also advancing by leaps and bounds. In the scenes of automobile, medicine, aerospace, and public service, we have fully enjoyed the convenience brought by industrial robots. However, with the continuous development of industrial robot-related concepts and technologies, human-computer interaction and cooperation have become the development trend of industrial robot. In this paper, the human-machine cooperation and path optimization of industrial robot in a complex road environment are studied and analyzed. At the theoretical modeling level, firstly, the industrial robot is modeled and obstacle avoidance is analyzed based on the kinematics of industrial robot; thus, an efficient and concise collision detection model of industrial robot is proposed. At the algorithm level, in view of the complex road conditions faced by industrial robots, this paper will study and analyze the obstacle avoidance strategy of humancomputer cooperation and real-time path optimization algorithm of industrial robots. Based on the virtual target point algorithm, this paper further improves the problem that the goal of the traditional path planning algorithm cannot be fully covered, so as to propose the corresponding improved path planning algorithm of industrial robots. In the experimental part, based on the existing industrial robot system, the human-machine cooperation and path planning system proposed in this paper are designed. The experimental results show that the algorithm proposed in this paper improves the accuracy of obstacle avoidance by about 10 points and the corresponding convergence speed by about $5 \%$ compared with the traditional algorithm and the experimental effect is remarkable.
\end{abstract}

\section{Introduction}

With the rapid development of industrial robots, more and more kinds of industrial robots come into people's daily life. Industrial robots can completely replace human beings to complete highly repetitive work. At the same time, they also further liberate productivity and improve production efficiency. However, with the continuous advancement of the concept of industrial robot and related technologies, the technology of industrial robot is developing in the direction of man-machine cooperation. The new man-machine cooperation mode will continue to break the boundaries between traditional industrial robots and workers, leading to the development trend of industrial robot technology [1-3]. The industrial robot based on human-computer cooperation makes it possible to cooperate with human beings without absolute isolation by safety fence, thus further reducing the distance between human and machine, reducing the area required by industrial production, fully combining the advantages of human and machine, and realizing complementary advantages. In the actual production activities, let the robot complete the mechanical work with high repeatability, and let the human complete the work with relatively strong flexibility, which needs continuous optimization by manual work $[4,5]$. However, compared with the traditional industrial robots, the development of humancomputer cooperation has brought about the security problems of the system. When human beings cooperate with machines and industrial robots move in the production place, the collision avoidance between human and machines, between machines and machines, and between machines and other objects can hardly be guaranteed. Industrial robot 
human-computer cooperation and corresponding path optimization algorithm has become an important research direction in the development of human-computer cooperation, and it is also the key difficulty to further promote this technology [6-8].

At present, the research on human-computer cooperation and path planning of industrial robot mainly focuses on the sensor level, which installs a large number of sensors on human and industrial robot, so as to realize mutual perception between industrial robot and human [9]. Sensor sensing of the current mainstream technology mainly used sensors to collect uncertain or unknown factors in the environment to capture human action. Through full analysis of the captured information, it generates the motion simulation representation of human and industrial robot and estimates human behavior by this simulation representation. Then, the actual distance between human and industrial robot is further judged, and the distance is summarized into the collision strategy of human and robot. At this time, when the corresponding collision risk is confirmed by industrial robot, the corresponding collision strategy will be activated, and the corresponding path trajectory of industrial robot will be generated, so as to realize the obstacle avoidance processing of human or related objects and machines [10-12]. In addition to the above-mentioned contact sensor technology, the current mainstream human-computer cooperation and path planning technology also includes contact motion capture system, but such systems often need to bear high operating costs when applied in actual scenes, and their customization degree is also high, which is not conducive to industrial promotion and use $[13,14]$. Based on this, this paper will study and analyze the human-machine cooperation and path optimization of industrial robot in a complex road environment. Firstly, the industrial robot is modeled and obstacle avoidance is analyzed based on the kinematics of the industrial robot, so as to put forward an efficient and concise human-machine collision detection model of industrial robot. In view of the complex road conditions faced by industrial robots, this paper will study and analyze the obstacle avoidance strategy of humancomputer cooperation and real-time path optimization algorithm of industrial robots. Based on the virtual target point algorithm, this paper further improves the problem that the goal of traditional path planning algorithm cannot be fully covered and puts forward the corresponding improved algorithm of industrial robot path planning [15]. In the experimental part, based on the existing industrial robot system, this paper designs the human-computer cooperation and path planning system. The experimental results show that the algorithm proposed in this paper can achieve the overall obstacle avoidance of industrial robot more efficiently than the traditional algorithm, and the planned path is more safe and efficient, which verifies the feasibility and superiority of the algorithm.

The structure of this paper is as follows: in the second section of this paper, the research status of human-computer cooperation and path planning algorithm of industrial robot is analyzed. The third section of this paper will focus on the optimization algorithm of human-computer cooperation and path planning of industrial robot, aiming at the research and analysis of human-computer cooperation and path optimization of industrial robot in a complex road environment. In the fourth section of this paper, the simulation system is designed based on the algorithm proposed in this paper, the simulation experiments are carried out, and the experimental results are analyzed. The summary is presented in the final section.

\section{Related Research: Research Status of Human- Computer Cooperation and Path Planning Algorithm of Industrial Robot}

At present, the essence of the research on human-computer cooperation and path planning algorithm of industrial robot is the safety of human-computer cooperation. Based on this, a large number of researchers and $R \& D$ institutions have carried out research and analysis on it. At the physical level, relevant researchers in the United States and Europe have proposed to use physical materials with absorption elasticity to develop industrial robots, so as to reduce the damage caused by the collision between robots and human bodies. The robot combines the nonlinear control theory with the electric drive system to realize the dynamic control of the industrial robot, so as to further ensure the safety of workers. With the continuous development of this kind of robot technology, the new robot has the characteristics of smooth structure, small size, and lightweight. However, this kind of robot does not completely solve the safety problem of human-computer cooperation, it only reduces the corresponding damage, and its corresponding production cost and maintenance cost are relatively high [16-18]; relevant scholars have studied and analyzed the control algorithm, and the main research scope is focused on obstacle avoidance strategy and path planning. Asian scholars have proposed a motion planning algorithm for industrial robots based on risk index minimization, which divides the robot motion path and region into safe path planning, online realtime safe trajectory planning, and real-time obstacle avoidance control [19, 20]; American scholars have designed a distributed distance sensor, which combines the risk assessment algorithm of the sensor with the control of industrial robot and then allows the robot to make corresponding obstacle avoidance actions, so as to improve the safety of the system; European scientists propose a motion control strategy to ensure safety, which mainly uses camera technology to monitor the working area of robot, calculates the distance between industrial robot and human in real time, and then defines various behaviors of robot based on this distance to ensure production efficiency and human safety [21, 22]; relevant researchers have focused on the application of video monitoring technology. Asian scientists used the somatosensory camera technology to track the position of workers, so as to obtain the three-dimensional coordinates of human joints. At the same time, they obtained the accurate position information of the target object through image processing technology and finally achieved a safe man-machine cooperation relationship [23]. 
Based on the somatosensory camera technology, Japanese scholars proposed further optimization of tracking technology. They developed an algorithm to estimate the distance between the omnipoint and the obstacle in Cartesian space and used the truncated cone generated by the pixels in the depth image to estimate the distance, so as to quickly calculate the distance between the industrial robot and human beings, so as to improve the running speed of the whole system $[24,25]$.

\section{Optimization Algorithm Analysis of Human- Machine Cooperation and Path Planning for Industrial Robot}

This section will mainly discuss and analyze the algorithm optimization of the current industrial robot human-machine cooperation and path planning level, which mainly includes the optimization of two core algorithms; the corresponding are the industrial robot kinematics optimization modeling analysis and the industrial robot human-machine cooperation security strategy and path planning research. The corresponding system framework diagram is shown in Figure 1. From the diagram, we can see that the ultimate goal of human-computer cooperation of industrial robot is to protect the personal safety of the collaborators. The corresponding diagram contains the algorithm hardware and software components of the system, as well as the security strategy of the whole system.

\subsection{Kinematics Optimization Modeling Analysis of Industrial} Robot. In order to better solve the problem of humancomputer cooperation and path planning of industrial robot, this section analyzes the kinematics modeling of industrial robot. In this paper, we focus on the axis robot as an example for analysis and research, its specific needs to go through the industrial robot position description, attitude description, and link description. In this paper, the collision strategy between industrial robot and human is considered in the actual modeling, and based on this, the kinematics optimization of industrial robot is carried out. The principle framework of the corresponding industrial robot kinematics model is shown in Figure 2.

In the corresponding position description part of the industrial robot, a certain space coordinate system is established based on three-dimensional space, and the points on the rigid body of the industrial robot are represented by three-dimensional Cartesian vector. In the corresponding orientation description level, the attitude representation is mainly based on the relative coordinates of the coordinate system. Assuming that $\{Q\}$ is corresponding to the original coordinate system, then the corresponding rigid body coordinate system corresponds to $\{P\}$, and the attitude of the rigid body can be expressed as the position of the coordinate system fixedly connected to the rigid body relative to the original coordinate system. In the description of the pose of the corresponding industrial robot, this paper focuses on its corresponding position and pose. At this time, the corresponding rigid body feature points such as the centroid or the center point are usually taken as the origin of the rigid body coordinate system. Compared with the original coordinate system $\{Q\}$, the corresponding rigid body coordinate system can be expressed as the following matrix 1, and the corresponding matrix has been homogeneous:

$$
Q_{A}^{B}=\left[\begin{array}{cccc}
p_{i} & o_{i} & p_{i} & o_{i} \\
p_{j} & o_{j} & p_{j} & o_{j} \\
p_{z} & o_{z} & p_{z} & o_{z} \\
0 & 0 & 0 & 1
\end{array}\right] .
$$

In the corresponding linkage description, it is mainly subject to the structure of industrial robot. The corresponding structure of conventional industrial robot is spatial open-chain structure. The corresponding connecting rod is processed in series through some column joints. The first link of corresponding industrial robot is recognized as the base of industrial robot by default, and the latter link of the corresponding group is regarded as the end actuator. The corresponding modeling rules of connecting rod parameters based on this rule are as follows:

(1) The corresponding $z$-axis direction in the corresponding linkage system coordinate system is set as the axis of the joint of industrial robot.

(2) The corresponding origin is assumed to be the vertical line corresponding to the $z$-axis direction.

(3) The corresponding industrial robot $x$-axis is set as the $z$-axis, and the vertical line of its adjacent $z$-axis and the corresponding direction is determined by $z$-axis.

(4) The setting of the corresponding $y$-axis follows the right-hand rule and is determined by the right-hand rule.

Based on the preparation of the above-mentioned related modeling, the collision strategy between industrial robot and human is considered and the kinematics model of industrial robot is optimized. In the actual modeling, the obstacles in the environment are regarded as operators in the collaborative environment, corresponding to the real-time updated obstacles. Aiming at the attitude and position information model of the industrial robot and human body established before, the precise three-dimensional model of industrial robot and human can be obtained. Based on the above model, the collision detection model is built based on the bounding box collision algorithm. The corresponding modeling methods are as follows:

(1) The collision detection of irregular obstacles in the environment is transformed into collision detection between corresponding regular geometry and can be calculated quickly.

(2) According to different types of bounding box algorithms, the corresponding envelope form is set as the AABB model.

(3) When the corresponding industrial robot envelope is collected, the images of multiple perspectives are 


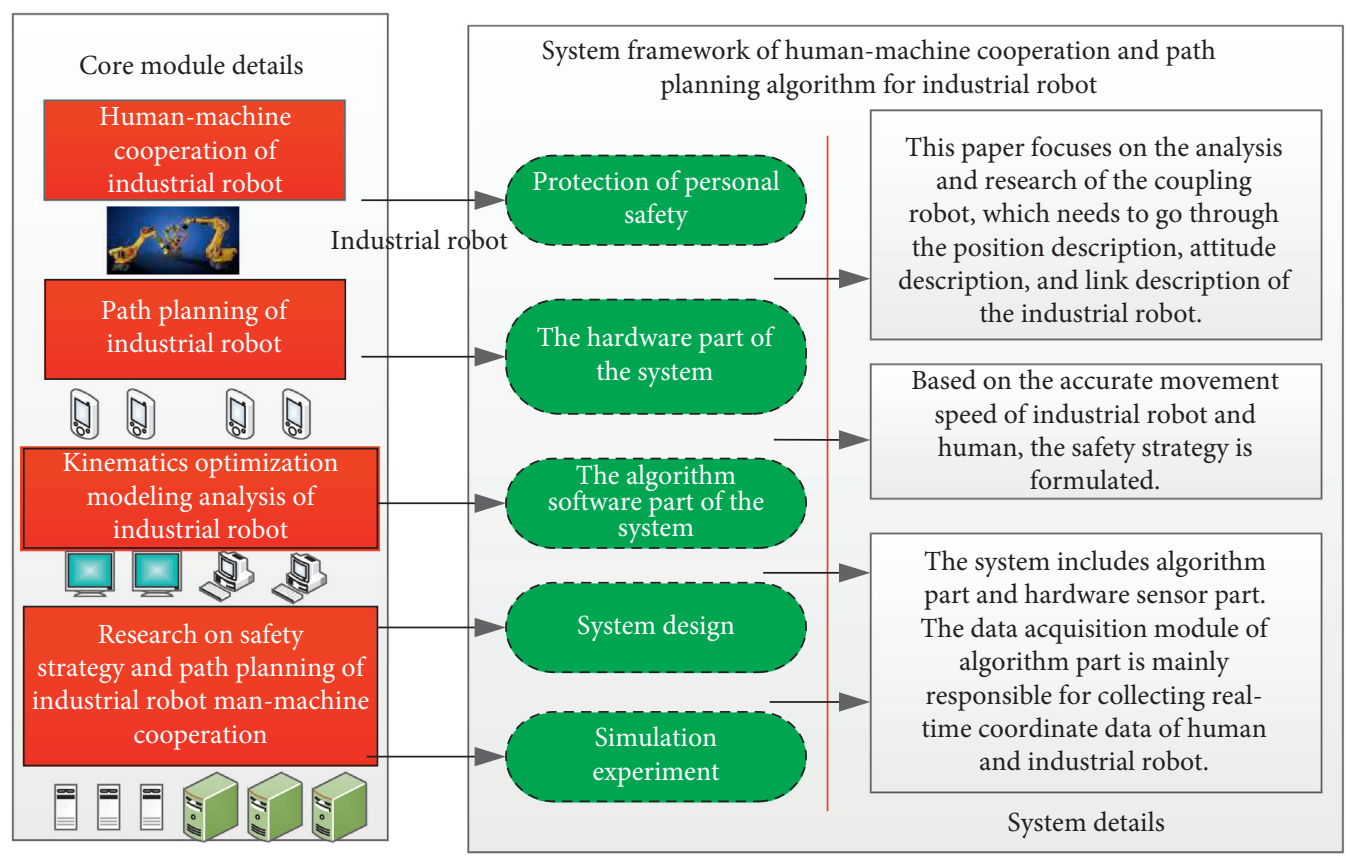

FIGURE 1: Framework of human-machine cooperation and path planning algorithm system for industrial robot.

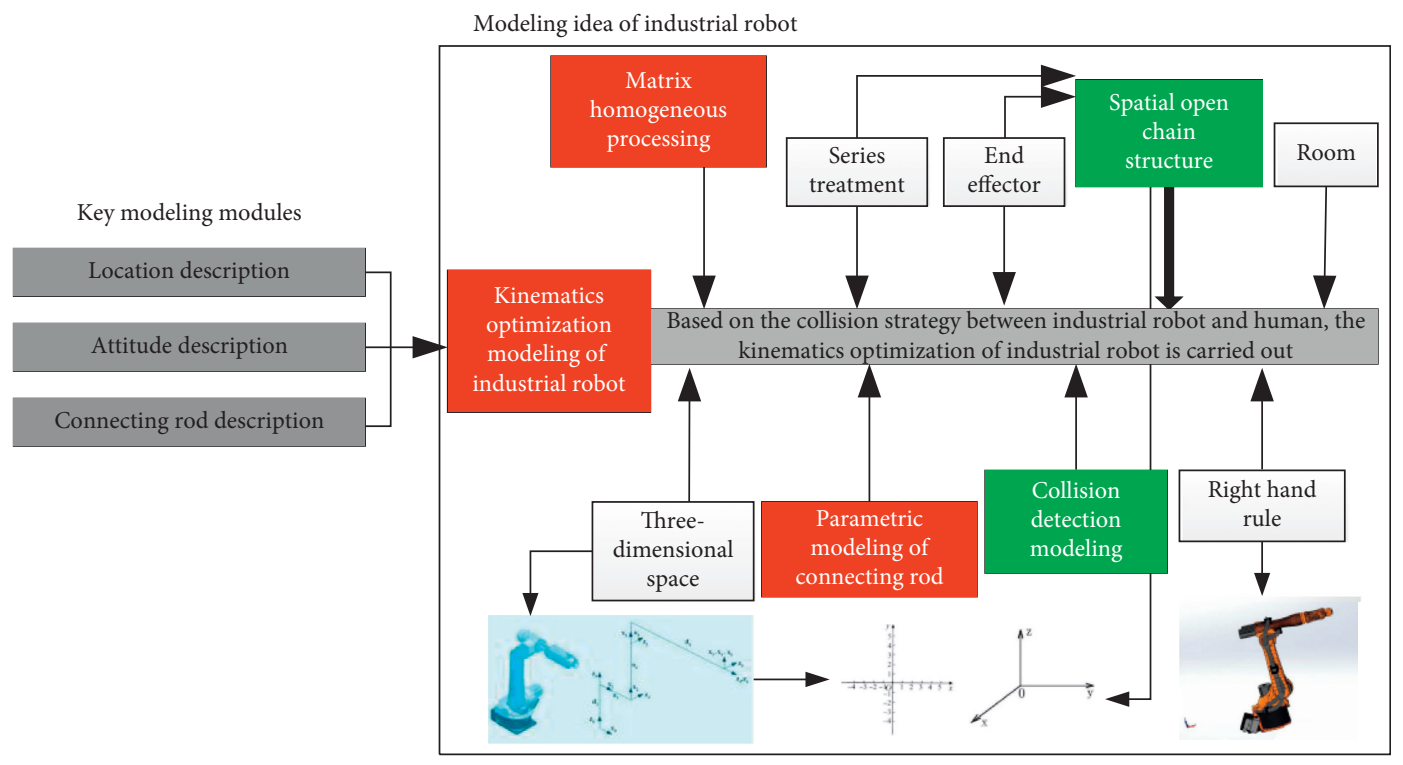

FIgURE 2: Schematic diagram of kinematic model of industrial robot.

collected, and the background is segmented and filtered, so as to get the segmented images of the robot from different perspectives.

(4) The corresponding space segmentation images are subdivided into several basic elements, and the position information of the finite cylinder and the corresponding key nodes of industrial robot are determined according to the known relevant information.

(5) A complete operational model of industrial robot is built.

The flowchart of the corresponding kinematic model of industrial robot is shown in Figure 3.
3.2. Analysis and Research on Safety Strategy and Path Planning of Industrial Robot Man-Machine Cooperation. Based on the above industrial robot kinematics modeling, considering the core algorithm of the whole system, namely, the safety strategy algorithm, this section will formulate the safety strategy based on the accurate movement speed of industrial robot and human. In the actual development of the corresponding safety strategy, the corresponding safety distance is set to $100 \mathrm{~cm}$. When the distance between the corresponding industrial robot and human exceeds the safety distance of $100 \mathrm{~cm}$, it indicates that the corresponding human is outside the working space of the industrial robot, and the corresponding industrial robot will work at a certain 


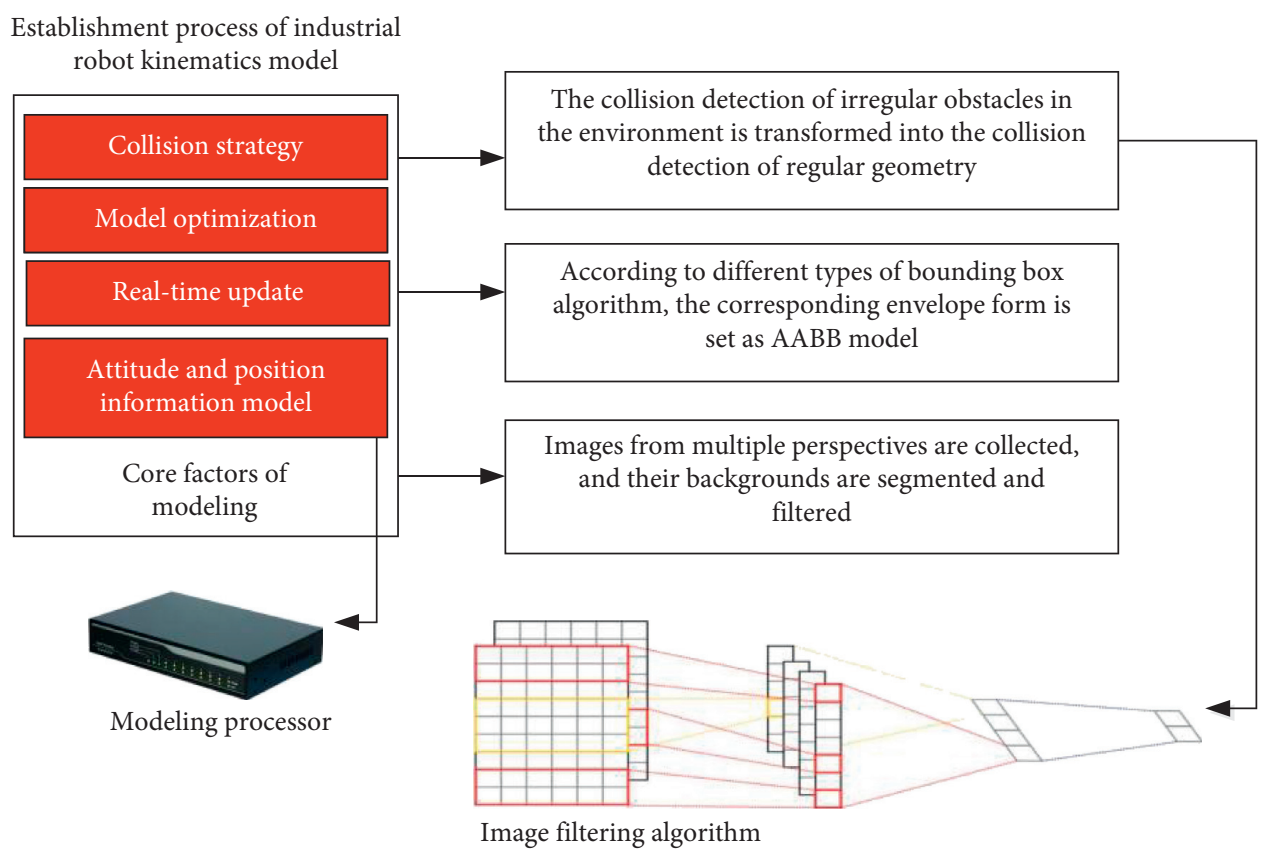

Figure 3: Flowchart of industrial robot kinematics model establishment.

speed $v$. When the safety distance between the corresponding industrial robot and human is less than $100 \mathrm{~cm}$, but this kind of data only has a single digit, then the strategy thinks that the data at this time is the noise data of the sensor, and the industrial robot still works at a certain speed $v$. When the corresponding warning data shows periodic stability, it is considered that the distance between the industrial robot and human is too close, and the working speed of the industrial robot is reduced. When the safety distance between the industrial robot and human is shrinking, the industrial robot stops working. The corresponding real-time adjustment function of the industrial robot is shown in formula (2). In the formula, $a$ represents the reduction percentage of the speed of the industrial robot, $d$ represents the safe distance between human and industrial robot, $d 1$ and $d 2$ represent the distance between human and machine when the industrial robot begins to reduce the working speed and stops working, and $V 1$ and $V 2$ represent the deceleration function of the industrial robot. To a certain extent, it controls the speed of industrial robots.

$$
a(d)=\left[\begin{array}{ccc}
0 & d>d \max & 0 \\
\frac{(1-v 1(d-15))}{y} d \min <d<d \max & v 1 \\
1 & d<d \min & v
\end{array}\right] .
$$

The most important of the above-mentioned industrial robot safety strategies is the precise control of the speed of industrial robots. In this paper, the auxiliary functions $V 1$ and $V 2$ are used to control the speed of industrial robots in practice. This can improve the security of the whole system and reduce the mechanical wear of the industrial robot. The corresponding speed precision control curve used in this paper is shown in Figure 4. From the figure, it can be seen that the safety of the system can be maximized by considering the corresponding speed, inertia, sharpness, and other attributes of industrial robots.

In the corresponding path planning algorithm level, we mainly consider improving the traditional form of artificial potential field. In this paper, the potential function is used to replace the traditional potential field force method for path planning of industrial robot, so as to achieve obstacle avoidance.

Through observation, analysis, and summary, the smaller the rotation angle of each joint of industrial robot is, the lower the probability of collision with human will be. Therefore, the improved gravitational potential function is shown in formula (3), where $k$ represents the coefficient of joint angle distance, $q$ represents the angle of each joint of industrial robot, and $p$ represents the position of the end point of industrial robot.

$$
M(p)=\left(\frac{k}{2}\right) *\left(\left\{p-p_{i}\right\}^{2}\right)+\left(\frac{k}{2}\right) k * \sum_{i=1}^{n}\left\{p-p_{i}\right\} .
$$

Aiming at the problem that the traditional path planning algorithm can not reach the target, this paper introduces the distance between the end point and the target point of the industrial robot on the basis of the gravitational potential function. When the corresponding industrial robot is close to the corresponding target point, the distance between the end point and the target point is shrinking, which plays a drag role on the corresponding repulsive potential field. Thus, the problem of unreachable target is solved. Based on this, the flowchart of the corresponding path planning algorithm is shown in Figure 5. From Figure 5, we can see that the corresponding details are as follows: 


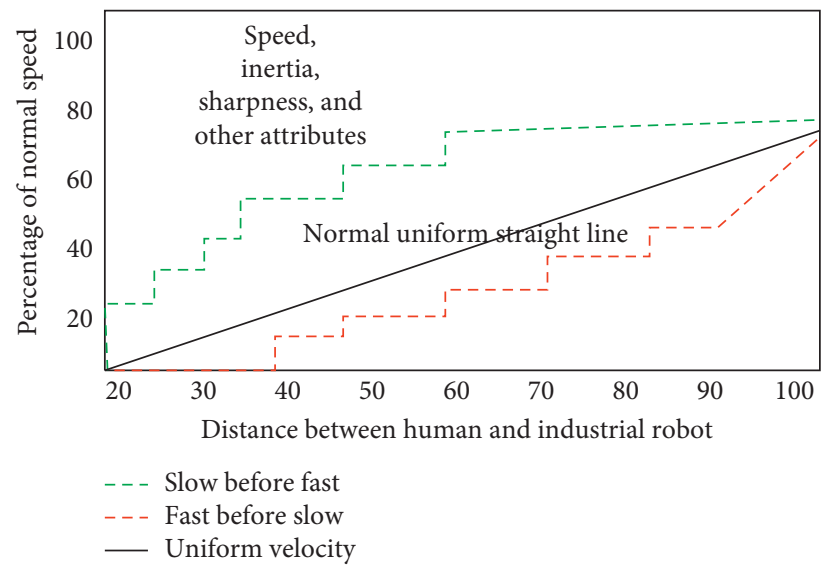

FIGURE 4: Speed precision control curve of industrial robot.

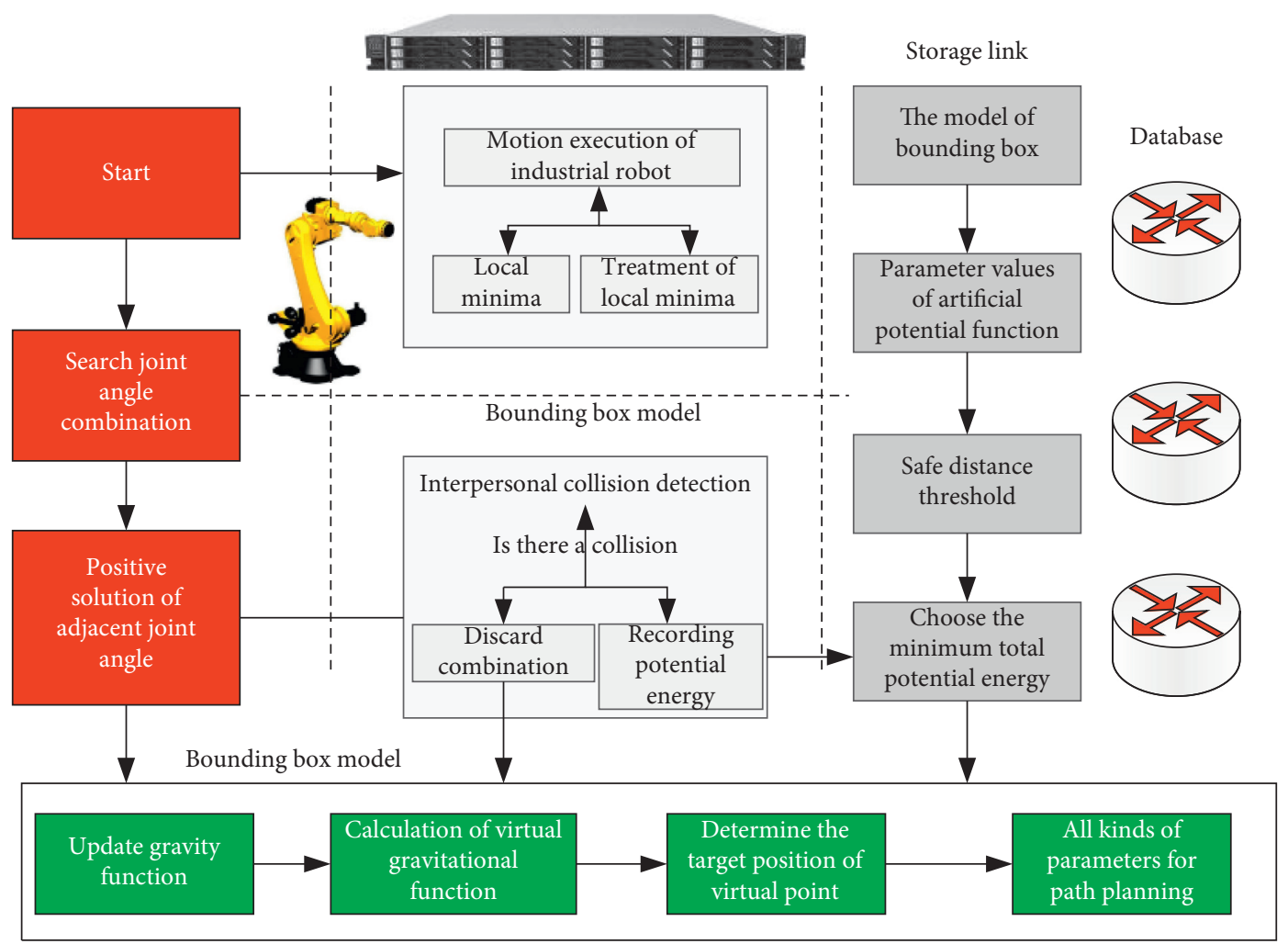

FIGURE 5: Flowchart of path planning algorithm for human-machine cooperation of industrial robot.

(1) Get the initial joint angle of the industrial robot, establish the bounding box model through the corresponding sensor, and determine the corresponding parameters of the improved artificial potential function, the repulsion influence distance, and the corresponding safety distance threshold.

(2) Combine the corresponding joints.

(3) Using the forward kinematics equation of the industrial robot, the coordinates of all joints and end points of the industrial robot under each joint combination are obtained

(4) Build the bounding box model and test the collision with the human bounding box.
(5) Substituting the recording distance corresponding to step 4 into the function formula of gravity and repulsion, the potential energy corresponding to each combination of industrial robot is calculated and compared, and the joint angle combined with the smallest total potential energy is selected.

(6) Path planning based on the above parameters.

\section{System Design and Simulation Experiment}

In order to verify the superiority of the algorithm, the overall design of the system is carried out first, and the corresponding overall design architecture is shown in Figure 6. It 
General principle of human-machine cooperation and path planning system for industrial robot

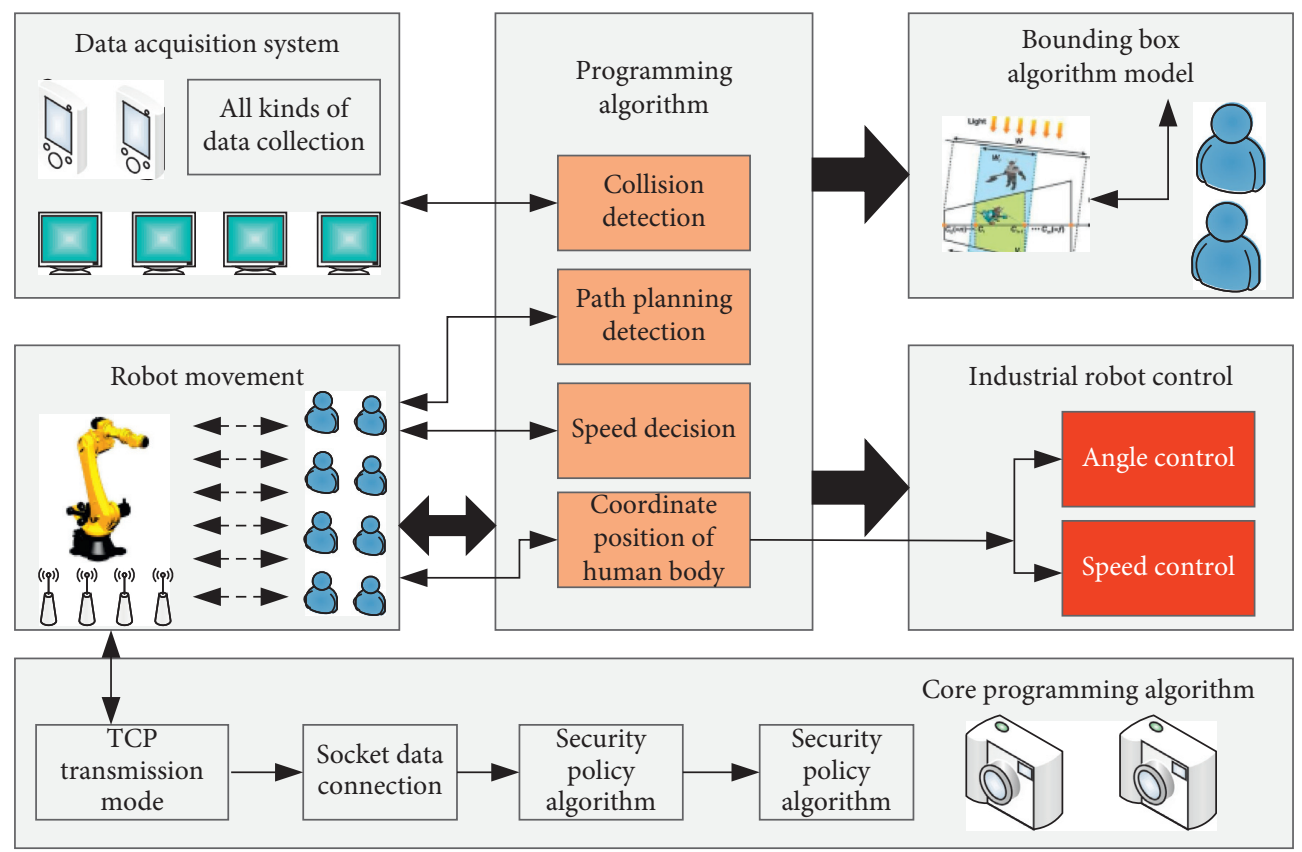

FigURE 6: General principle framework of industrial robot human-machine cooperation and path planning system.

can be seen from the figure that the corresponding system includes the algorithm part and the hardware sensor part. The data acquisition module corresponding to the algorithm part is mainly responsible for collecting the real-time motion coordinate data of human and industrial robot. The corresponding network transmission is mainly responsible for the data transmission between each module. The corresponding planning algorithm is mainly responsible for collision detection between industrial robot and human, and it also needs to further calculate the next motion angle and speed of industrial robot in this case. The corresponding real-time calculation data in the core control module will be verified by the virtual simulation and robot control module, and the real-time motion control of industrial robot will be completed.

The corresponding hardware module design level mainly includes five hardware modules, which are data acquisition module, network data transmission module, core algorithm module, virtual simulation module, and robot control movement module. In the corresponding data acquisition module, it mainly collects the spatial coordinate information of industrial robot and human movement and sends the data collected to the corresponding analysis module of the algorithm. The corresponding data needs to be cached in the corresponding local species after the acquisition. In the actual transmission, the latest data is obtained from the local at a certain time and transmitted to the collision avoidance path planning program for processing. In the corresponding network data transmission module, it is mainly responsible for the transmission of data in each module of the system, so as to ensure the reliability of data. The corresponding transmission mode adopts TCP transmission mode and socket to connect different data, so as to realize the reliable transmission of data. The corresponding algorithm module includes security strategy algorithm and path planning algorithm. It calculates the joint angle combination and corresponding motion speed of the next motion of industrial robot continuously and carries out real-time path planning processing based on the current corresponding data. At the corresponding speed control level of industrial robots, computer control line is mainly used to connect with the corresponding speed controller of industrial robot. The corresponding working flowchart of industrial robot control module is shown in Figure 7.

Based on the design of the above system, this paper carries out experimental simulation based on an industrial robot in the corresponding scene. The main simulation experiments of this paper include security strategy simulation and path planning simulation.

4.1. Security Policy Simulation Experiment. In order to further verify the obstacle avoidance ability of the industrial robot in the presence of environmental interference, on the premise that other control environment variables remain unchanged, observe the curve change of the end point of the industrial robot in the $z$-axis of the spatial coordinate in the interference and noninterference environment, and the corresponding change trajectory is shown in Figure 8. It can be seen from Figure 8 that, in the noninterference environment, the industrial robot has been moving along the direction corresponding to the $z$-axis and finally reaches the end point. In the corresponding interference environment, the industrial robot starts to avoid obstacles when it moves to the 34th step, and its corresponding joint conversion angle is also smaller and smaller. After successfully 


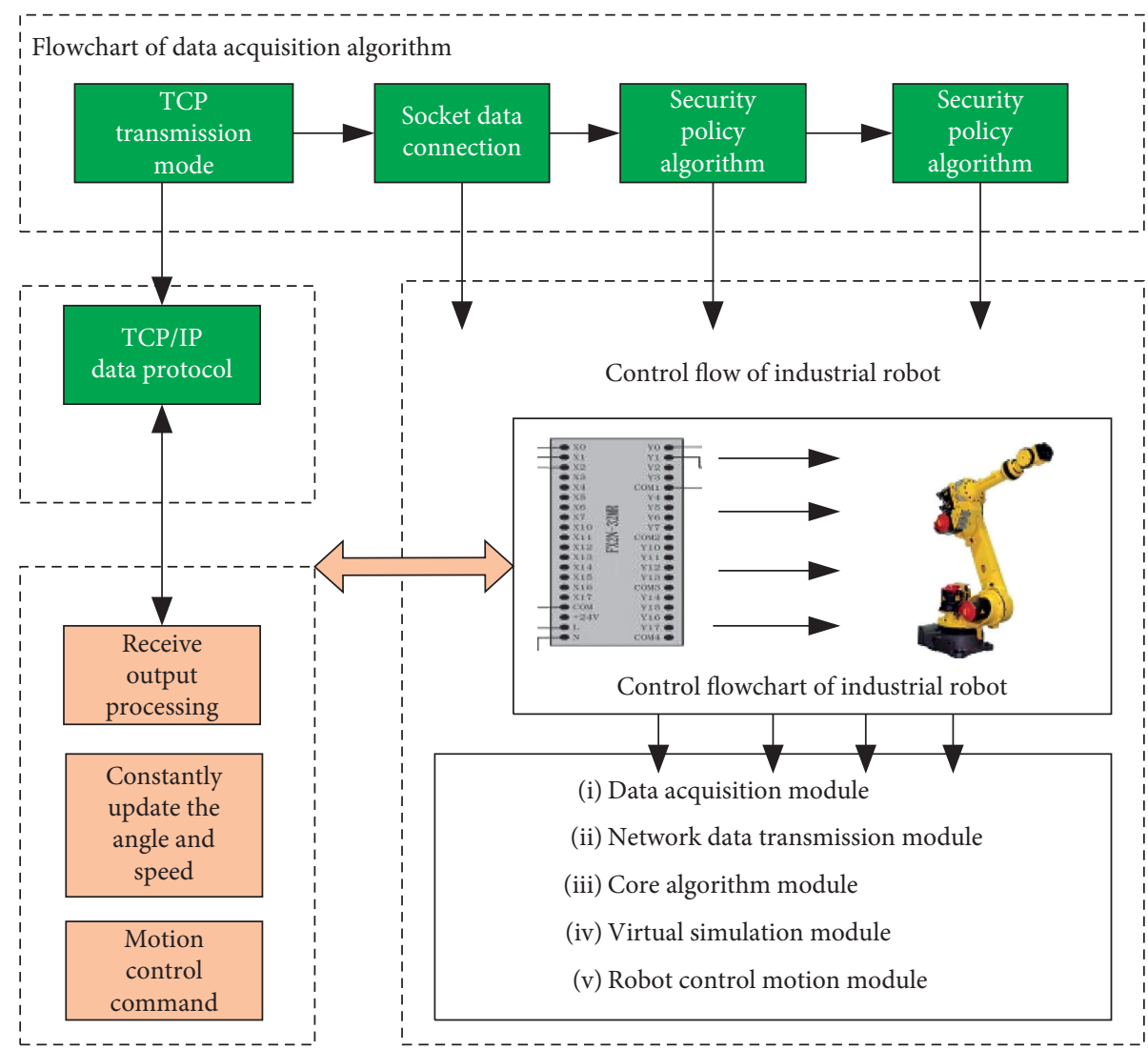

FIGURE 7: Working flow principle frame diagram of industrial robot control module.

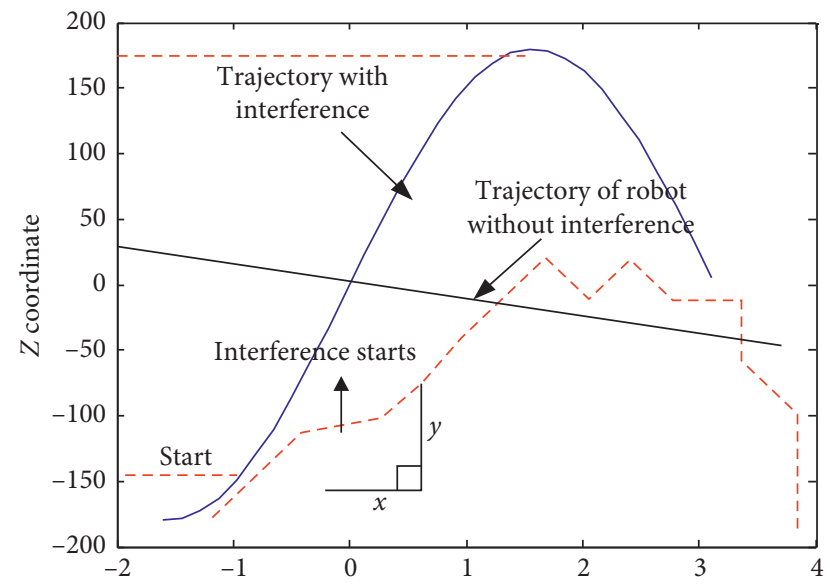

FIGURE 8: Corresponding state trajectory of industrial robot with and without interference.

bypassing the obstacles, it moves to the end point again. The corresponding security strategy simulation is mainly the obstacle avoidance experiment. When the distance between human and machine is less than $150 \mathrm{~mm}$, the corresponding industrial robot will actively avoid obstacles. The corresponding industrial robot reaches the specified target location after 166 times and 115 times, respectively. There is human interference process in the corresponding environment. From the analysis of the figure, we can see that the corresponding industrial robot motion trajectory is smooth and continuous, which proves that the algorithm in this paper is effective and feasible in the actual industrial robot motion to a certain extent.

Simulation of the robot trajectory in the case of interference in the above experiment is corresponding threedimensional obstacle avoidance diagram of industrial robot. From the figure, it can be seen that the industrial robot can effectively deal with the corresponding obstacles in threedimensional obstacle avoidance, and the corresponding curve is relatively smooth, which further verifies the effectiveness of the safety strategy algorithm in this paper.

4.2. Path Planning Simulation Experiment. In order to verify the advantages of the proposed algorithm in the path planning of industrial robots, the paper compares the algorithm with the traditional algorithm in the presence of interference. The main comparison indexes include the algorithm running time, the length of the algorithm planning path, and the security comparison of the algorithm. The corresponding algorithm average running time comparison curve is shown in Figure 9(a). From the figure, it can be seen that the algorithm time proposed in this paper is relatively short compared with the other two path planning algorithms, the corresponding real-time effect is better, and the path adjustment can be performed in real time when the path planning is carried out. Figure 9(b) shows the curve of the algorithm and the other two traditional algorithms in the length of the algorithm planning path. From the graph, it can 


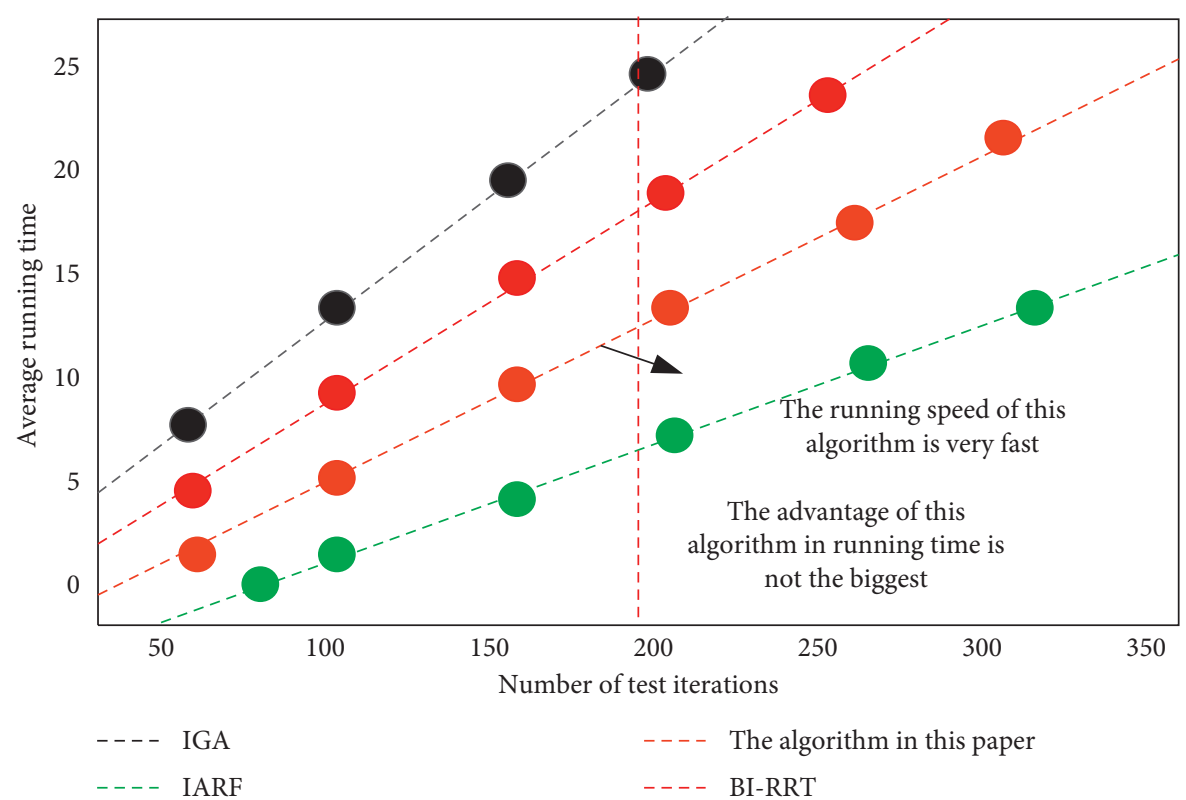

(a)

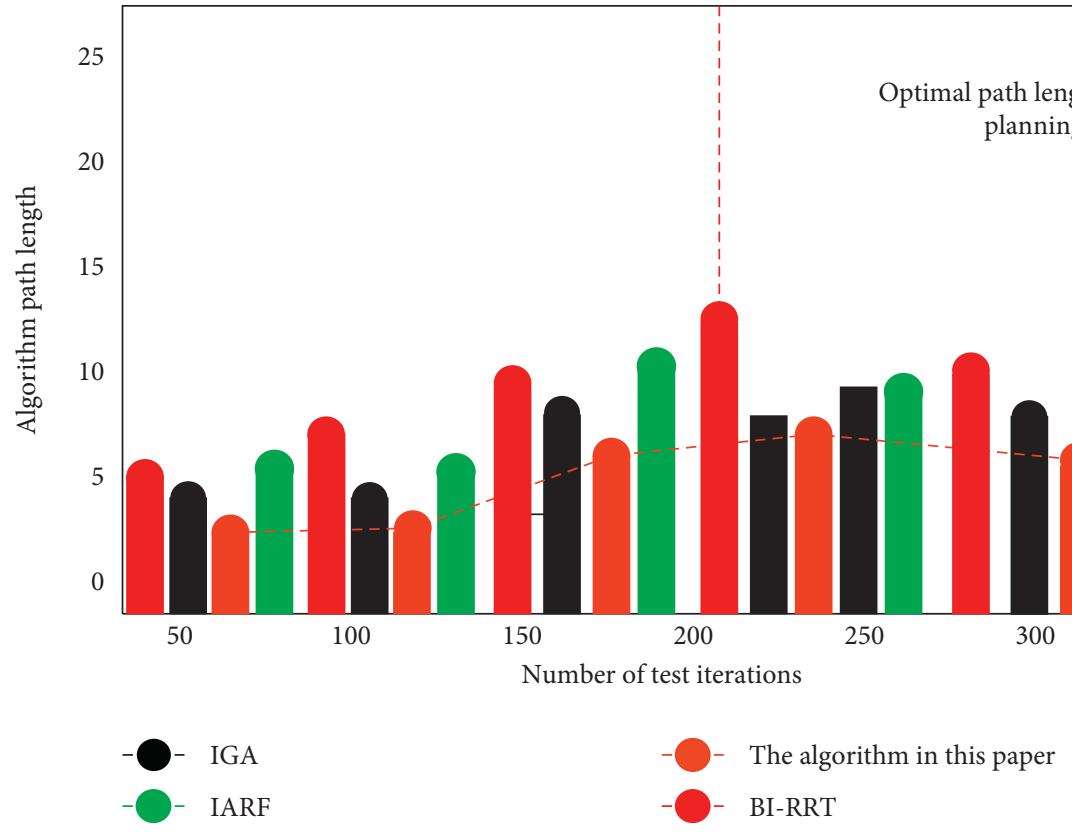

(b)

Figure 9: Continued. 


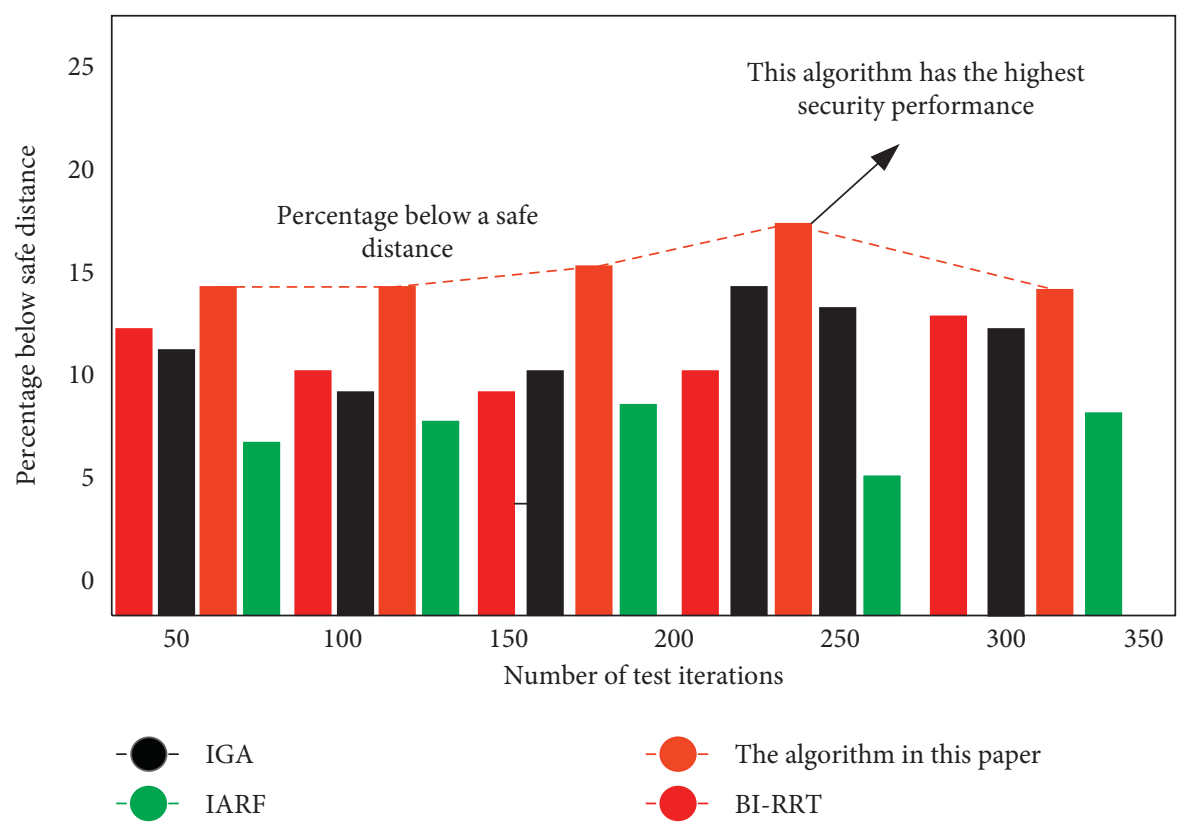

(c)

Figure 9: (a) Comparison curve of average running time of algorithm. (b) Comparison curve of path length of algorithm planning. (c) Algorithm security comparison curve.

be seen that the corresponding planning path of the algorithm proposed in this paper is the shortest and the corresponding algorithm is stable. Although the traditional algorithm has some advantages in time, the corresponding planning path is longer. Figure 9(c) shows the contrast curve of the algorithm and the traditional algorithm in the algorithm security. From the figure, it can be seen that the proposed algorithm can ensure that the distance between the links and obstacles of industrial robots is greater than the safety distance compared with the traditional algorithm, while the distance corresponding to other traditional algorithms will be less than the safety distance. This will cause the collision between industrial robot and obstacles or human beings, which will affect the safety strategy of the whole system.

Based on the above experiments and analysis, it can be concluded that the algorithm in this paper has obvious advantages compared with the traditional algorithm in the aspect of human-computer cooperation and path planning of industrial robot, and its corresponding obstacle avoidance effect is significant, which has high practical promotion value.

\section{Summary}

This paper mainly analyzes the development trend of industrial robot and the research hotspots of human-computer cooperation and path planning and gives the development difficulties of human-computer cooperation and path planning of industrial robot. In view of the above difficulties, this paper studies and analyzes the human-machine cooperation and path optimization of industrial robot in a complex road environment. Firstly, based on the kinematics of industrial robot, the theoretical modeling and obstacle avoidance analysis of industrial robot are carried out; thus, an efficient and concise human-machine collision detection model of industrial robot is proposed. In view of the complex road conditions faced by industrial robots, this paper studies and analyzes the obstacle avoidance strategy of human-computer cooperation and real-time path optimization algorithm of industrial robots. Based on the virtual target point algorithm, it further improves the problem that the traditional path planning algorithm can not fully cover the target and puts forward the corresponding improved path planning algorithm of industrial robots. In the experimental part, based on the existing industrial robot system, this paper designs the human-computer cooperation and path planning system. The experimental results show that the algorithm proposed in this paper can achieve the overall obstacle avoidance of industrial robot more efficiently than the traditional algorithm, and the planned path is more safe and efficient, which verifies the feasibility and superiority of the algorithm. In the following research, this paper will focus on the application of the proposed algorithm in large-scale industrial scenes and study the corresponding algorithm consumption and convergence problems.

\section{Data Availability}

Relevant data can be obtained from the corresponding author upon request.

\section{Conflicts of Interest}

The author declares that there are no conflicts of interest. 


\section{References}

[1] F. Mars, M. Deroo, and J.-M. Hoc, "Analysis of humanmachine cooperation when driving with different degrees of haptic shared control," IEEE Transactions on Haptics, vol. 7, no. 3, pp. 324-333, 2014.

[2] Z. Yahouni, N. Mebarki, F. Belkadi, A. Shahzad, and A. Bernard, "Human-machine cooperation in planning and scheduling: a case study on an unstable environment," $E u$ ropean Journal of Industrial Engineering, vol. 12, no. 6, pp. 757-764, 2018.

[3] L. Athanasopoulou, A. Papacharalampopoulos, P. Stavropoulos, and D. Mourtzis, "Design and manufacturing of a smart mobility platform's context awareness and path planning module: a PSS approach," Procedia Manufacturing, vol. 51, no. 1-2, pp. 61-66, 2020.

[4] X. L. Jia, X. Q. Yang, and Y. S. Deng, "Path planning of multirobot fish based on cooperative game model," Applied Mechanics and Materials, vol. 341-342, no. 3, pp. 940-944, 2013.

[5] E. Masehian, M. Jannati, and T. Hekmatfar, "Cooperative mapping of unknown environments by multiple heterogeneous mobile robots with limited sensing," Robotics and Autonomous Systems, vol. 87, no. 6, pp. 188-218, 2017.

[6] J. Yang, D. Wang, B. Fan et al., "Online absolute pose compensation and steering control of industrial robot based on six degrees of freedom laser measurement," Optical Engineering, vol. 56, no. 3, pp. 034111.1-034111.9, 2017.

[7] Y. Chao, X. Chen, and N. Xiao, "Deep learning-based graspdetection method for a five-fingered industrial robot hand," IET Computer Vision, vol. 13, no. 1, pp. 61-70, 2018.

[8] R. Martínez and J. Carlos, "A function block based approach using assembly features for controlling virtual and real industrial robots," The Journal of Symbolic Logic, vol. 30, no. 2, 248-249, 2015.

[9] S. S. Pchelkin, A. S. Shiriaev, A. Robertsson et al., "On orbital stabilization for industrial manipulators: case study in evaluating performances of modified $\mathrm{PD}+$ and inverse dynamics controllers," IEEE Transactions on Control Systems Technology, vol. 11, no. 4, pp. 1-17, 2017.

[10] A. Ferrara and G. P. Incremona, "Design of an integral suboptimal second-order sliding mode controller for the robust motion control of robot manipulators," IEEE Transactions on Control Systems Technology, vol. 23, no. 6, pp. 2316-2325, 2015.

[11] M. P. Polverini, S. Formentin, L. Merzagora et al., "Mixed data-driven and model-based robot implicit force control: a hierarchical approach," IEEE Transactions on Control Systems Technology, vol. 8, no. 99, pp. 1-14, 2019.

[12] V. Nguyen, J. Johnson, and S. Melkote, "Active vibration suppression in robotic milling using optimal control," International Journal of Machine Tools and Manufacture, vol. 152, no. 5, Article ID 103541, 2020.

[13] J. Baek, W. Kwon, B. Kim, and S. Han, "A widely adaptive time-delayed control and its application to robot manipulators," IEEE Transactions on Industrial Electronics, vol. 66, no. 7, pp. 5332-5342, 2019.

[14] B. Xiao, H. K. Lam, G. Song, and H. Li, "Output-feedback tracking control for interval type-2 polynomial fuzzy-modelbased control systems," Neurocomputing, vol. 242, no. 12, pp. 83-95, 2017.

[15] H. Hassan, C. Dominguez, J.-M. Martinez, A. Perles, J.-V. Capella, and J. Albaladejo, "A multidisciplinary PBL robot control project in automation and electronic engineering," IEEE Transactions on Education, vol. 58, no. 3, pp. 167-172, 2015.

[16] T. Tsuji, J. Ohkuma, and S. Sakaino, "Dynamic object manipulation considering contact condition of robot with tool," IEEE Transactions on Industrial Electronics, vol. 63, no. 3, pp. 1972-1980, 2016.

[17] J. Qin, S. Wang, Y. Kang, and Q. Li, "Circular formation algorithms for multiple nonholonomic mobile robots: an optimization-based approach," IEEE Transactions on Industrial Electronics, vol. 15, no. 5, pp. 22-32, 2018.

[18] J. Edwards, "Signal processing helps put robot users in control special reports," IEEE Signal Processing Magazine, vol. 33, no. 3, pp. 8-11, 2016

[19] J. Yu, W. Chen, and G. Xie, "Coordination of multiple robotic fish with applications to underwater robot competition," IEEE Transactions on Industrial Electronics, vol. 63, no. 2, pp. 1-12, 2015.

[20] P. Lu, W. Yu, G. Chen, and X. Yu, "Leaderless consensus of ring-networked mobile robots via distributed saturated control," IEEE Transactions on Industrial Electronics, vol. 67, no. 12, pp. 10723-10731, 2020.

[21] S. Rahman and R. Ikeura, "Weight-prediction-based predictive optimal position and force controls of a power assist robotic system for object manipulation," IEEE Transactions on Industrial Electronics, vol. 63, no. 9, pp. 11-19, 2016.

[22] X. Li and C. C. Cheah, "A simple trapping and manipulation method of biological cell using robot-assisted optical tweezers: singular perturbation approach," IEEE Transactions on Industrial Electronics, vol. 64, no. 2, pp. 1656-1663, 2017.

[23] C. Faria, C. Vale, T. Machado et al., "Experiential learning of robotics fundamentals based on a case study of robot-assisted stereotactic neurosurgery," IEEE Transactions on Education, vol. 59, no. 2, pp. 45-51, 2016.

[24] A. H. Memar and E. T. Esfahani, "A robot gripper with variable stiffness actuation for enhancing collision safety," IEEE Transactions on Industrial Electronics, vol. 67, no. 8, pp. 6607-6616, 2020.

[25] H.-K. Shin and B. K. Kim, "Energy-efficient gait planning and control for biped robots utilizing vertical body motion and allowable ZMP region," IEEE Transactions on Industrial Electronics, vol. 62, no. 4, pp. 2277-2286, 2015. 